\title{
Propuesta para el manejo de la coagulopatía asociada a COVID-19 en niños
}

\section{Proposal for the management of COVID-19-associated coagulopathy in children}

\author{
Susana Palomino-Nolasco ${ }^{*}$, Gerado M. Pacheco-González y Claudia V. Saldaña-Díaz ${ }^{2}$ \\ ${ }^{1}$ Facultad de Medicina, Universidad Privada San Juan Bautista; ${ }^{2}$ Unidad Funcional de Investigación Nacional Materno Perinatal. Lima, Perú
}

Estimado editor,

Luego de haber revisado el artículo escrito por Ávila, et al. «Propuestas para el manejo de la coagulopatía asociada a COVID-19 en niños» consideramos resaltar en tres puntos las complicaciones trombóticas por enfermedad por coronavirus 2019 (COVID-19) asociada a síndrome de dificultad respiratoria aguda que requiere de una estrategia terapéutica anticoagulante.

- La inflamación pulmonar genera daño de la vasculatura pulmonar y desencadena una trombosis seguida de un estado de hipercoagulabilidad que se manifiesta con trombocitopenia y niveles elevados de fibrinógeno y dímero $\mathrm{D}^{1}$.

- El coronavirus 2 del síndrome respiratorio agudo grave (SARS-CoV-2) activa el sistema fibrinolítico de forma anormal, ya que los depósitos de fibrina intrapulmonar se recambian a una fibrina anormal responsable de la coagulopatía ${ }^{2}$.

- Es necesario una anticoagulación profiláctica con enoxaparina, ya que en niños y adolescentes se ha descrito un síndrome semejante a la enfermedad de Kawasaki ${ }^{3}$.

Las complicaciones trombóticas y el daño pulmonar por la infección por SARS-CoV-2 se relacionan, por lo que se requiere un manejo profiláctico anticoagulante.

\section{Financiamiento}

Los autores declaran que no existió financiamiento.

\section{Conflicto de intereses}

Los autores declaran que no existe conflicto de intereses.

\section{Bibliografía}

1. Wool GD, Miller JL. The Impact of COVID-19 Disease on Platelets and Coagulation. Pathobiology. 2021;88(1):15-27.

2. Levi M, Thachil J. Coronavirus Disease 2019 Coagulopathy: Disseminated Intravascular Coagulation and Thrombotic Microangiopathy-Either, Neither, or Both. Semin Thromb Hemost. octubre de 2020;46(07):781-4.

3. Cervera Ubierna A. Tratamiento de la infección por SARS-CoV-2. Acta Pediátrica México. 29 de junio de 2020;41(4S1):121. CC BY-NC-ND (http://creativecommons.org/licenses/by-nc-nd/4.0/). 